\title{
Using gridCoal to assess whether standard population genetic theory holds in the presence of spatio-temporal heterogeneity in population size.
}

\author{
Barbora Trubenova ${ }^{1}$, Enikő Szép ${ }^{2}$, and Katalin Csillery ${ }^{3}$ \\ ${ }^{1} \mathrm{ETH}$ Zürich \\ ${ }^{2}$ IST Austria \\ ${ }^{3} \mathrm{WSL}$
}

February 24, 2022

\begin{abstract}
Spatially explicit population genetic models have long been developed, yet have rarely been used to test hypotheses about the spatial distribution of genetic diversity or the expected neutral levels of genetic divergence between populations. Here, we use spatially explicit coalescence simulations to explore the properties of the island model and the two-dimensional stepping stone model under a wide range of scenarios with spatio-temporal variation in deme size. We avoid the simulation of genetic data, using the fact that under the studied models, summary statistics of genetic diversity and divergence between demes can be approximated from coalescence times. We perform the simulations using gridCoal, a flexible spatial wrapper for the software msprime developed herein. In gridCoal, deme sizes can change arbitrarily across space and time, and migration rates between individual demes can be specified. We identify the different factors that can cause a deviation from the theoretical expectations, such as the simulation time in comparison to the effective deme size and the spatio-temporal autocorrelation across the grid. Our results highlight that Fst, a measure of the strength of population structure, principally depends on recent demography, which makes it robust to temporal variation in deme size. We also warn that predicting genetic diversity from coalescence times requires a much longer run time than needed for the estimation of Fst. Finally, we illustrate the use of gridCoal on a real-world example, the range expansion of silver fir (Abies alba Mill.) since the Last Glacial Maximum, using different degrees of spatio-temporal variation in deme size.
\end{abstract}

\section{Hosted file}

GridCoal_ResubmissionFinal.pdf available at https://authorea.com/users/462239/articles/ 557721-using-gridcoal-to-assess-whether-standard-population-genetic-theory-holds-in-thepresence-of-spatio-temporal-heterogeneity-in-population-size 

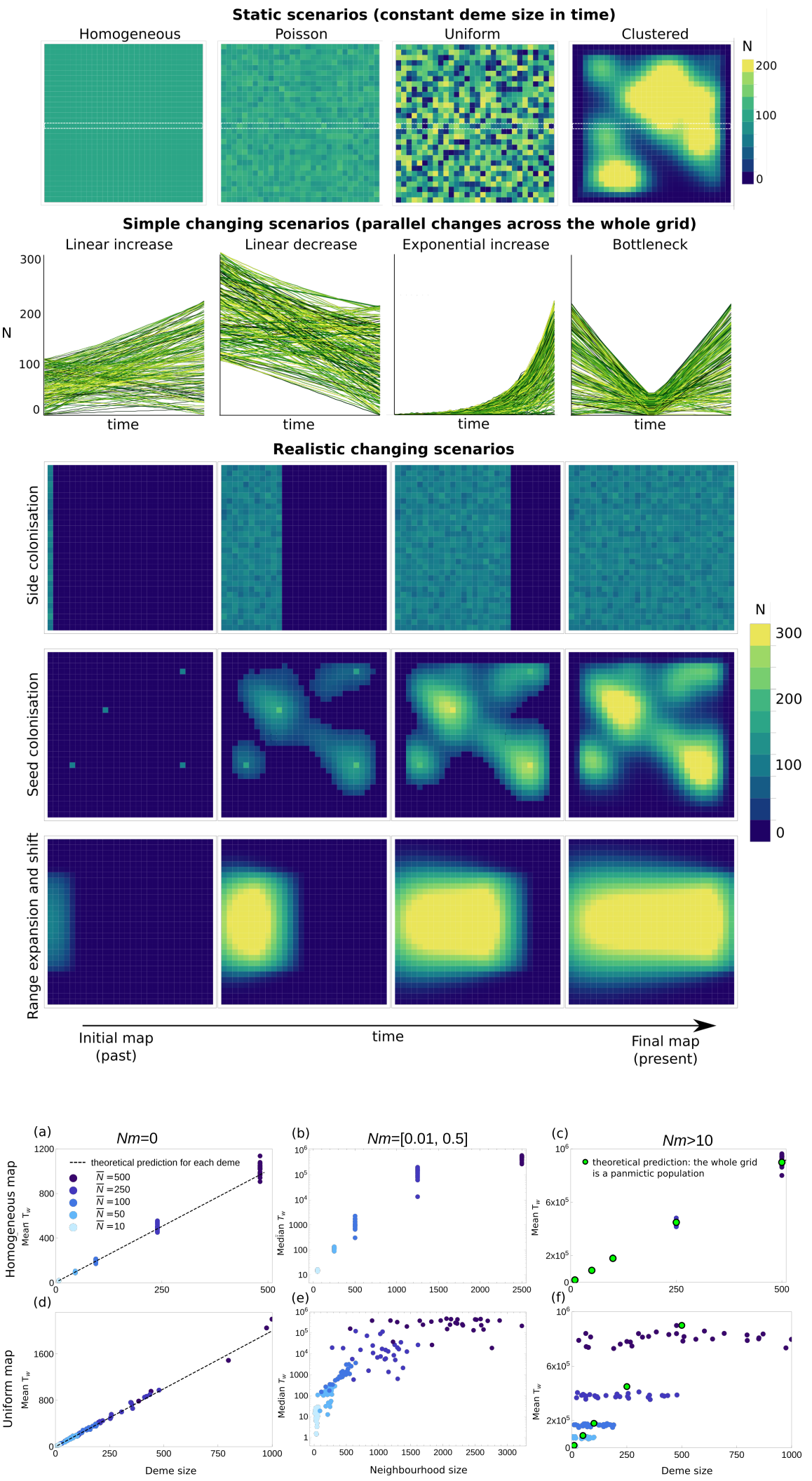

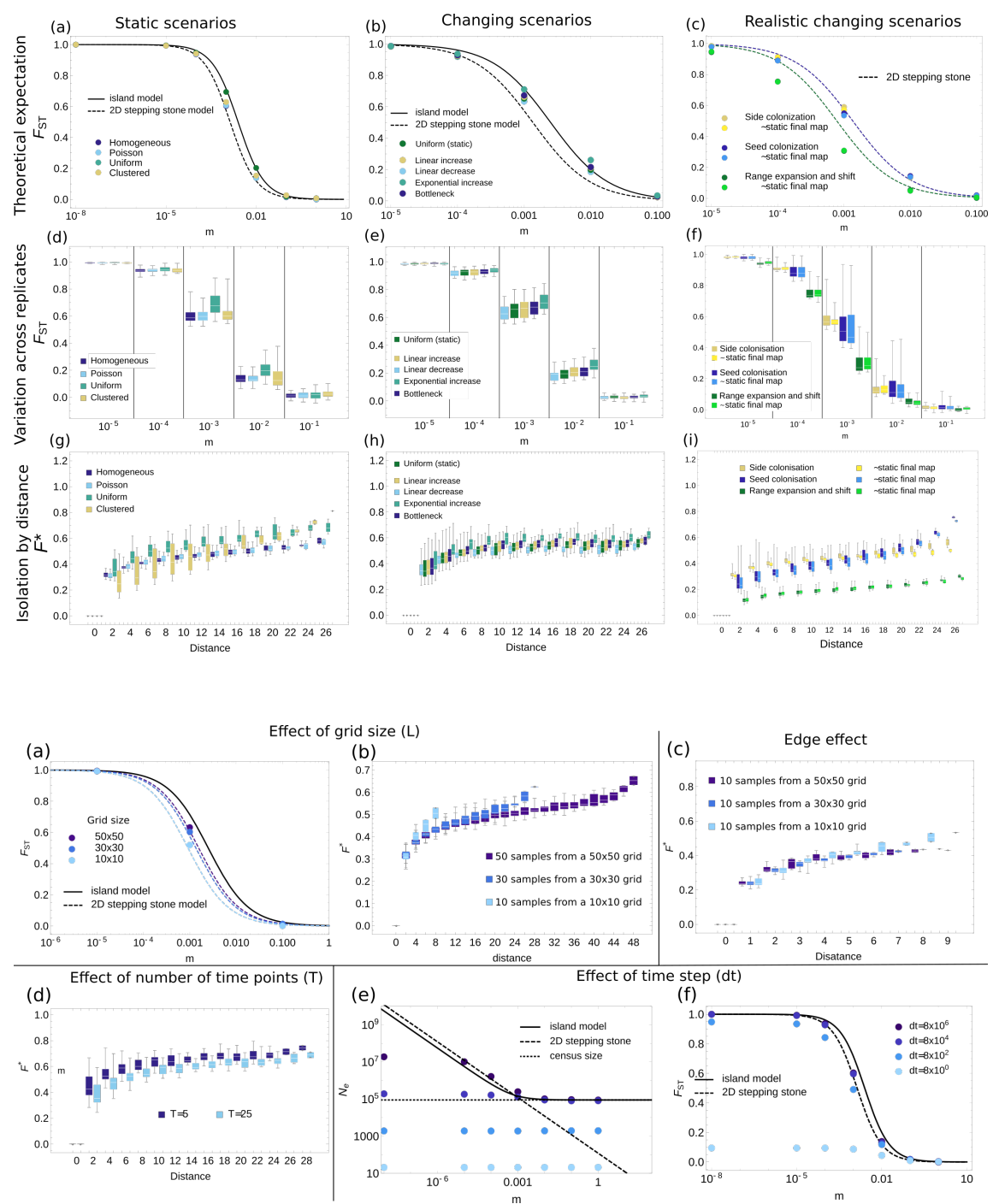


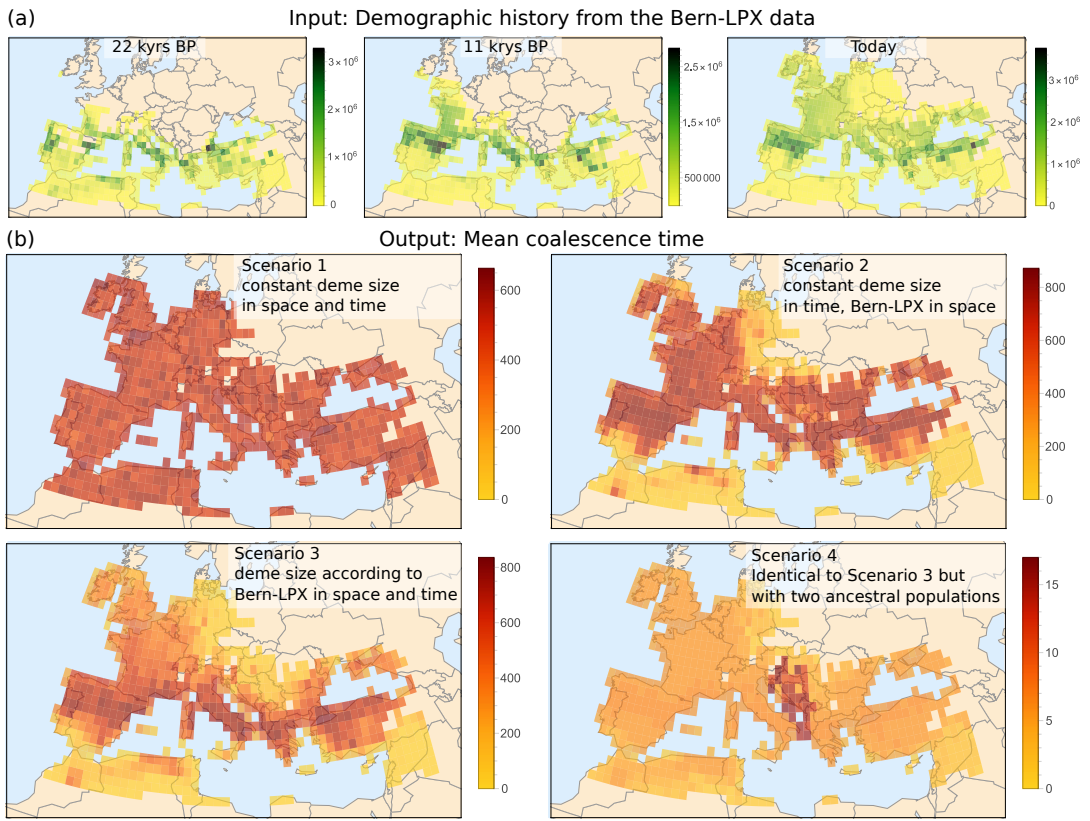

